Meta

Journal des traducteurs

Translators' Journal

\title{
Des faux amis chez nos cousins
}

\section{Pierre Mimin}

Volume 19, numéro 3, septembre 1974

URI : https://id.erudit.org/iderudit/003653ar

DOI : https://doi.org/10.7202/003653ar

Aller au sommaire du numéro

Éditeur(s)

Les Presses de l'Université de Montréal

ISSN

0026-0452 (imprimé)

1492-1421 (numérique)

Découvrir la revue

Citer cet article

Mimin, P. (1974). Des faux amis chez nos cousins. Meta, 19(3), 134-137.

https://doi.org/10.7202/003653ar d'utilisation que vous pouvez consulter en ligne.

https://apropos.erudit.org/fr/usagers/politique-dutilisation/ 


\section{Des faux amis chez nos cousins}

J'imagine que les traducteurs spécialistes, comme les modestes traducteurs secrétaires des chefs d'entreprise au Canada, rencontrent une difficulté singulière quand ils s'adressent à des francophones hors du Québec et surtout à ceux de la France hexagonale dont je suis citoyen. S'agissant, par exemple, d'opérations électorales, le traducteur, s'il avait eu affaire à ses compatriotes québécois, aurait été amené à parler du «jour de la nomination des candidats», expression comprise de tous les électeurs du lieu. Mais, écrivant à des étrangers, il sera pénétré du devoir de transformer cette expression, calquée de l'anglais «nomination day » en celle-ci : «jour de la présentation des candidats» qui, dans un français universel, s'accorde avec l'événement de type américain.

Combien d'hypothèses où le traducteur hésitera entre son désir d'être apprécié par son auditoire ordinaire et son devoir de surmonter et de proscrire l'usage des mauvais canadianismes résultant, comme dans mon exemple, de la traduction littérale du mot anglais par le mot français de même forme, ici le mot «nomination» qui n'a pas du tout le même sens dans l'alliance de mots « jour de la nomination des candidats $\gg$ en français et en anglo-américain.

Car s'il y a de mauvais canadianismes imposés par la conquête britannique puis par l'hégémonie économique des États-Unis de l'Amérique du Nord, il y en a d'issus tant de faits naturels, dispositions géographiques, vêtements, mœurs, traditions hérités de l'occupation iroquoise ou de la période française depuis Jacques Cartier jusqu'à Montcalm, canadianismes utiles qu'il faut conserver (ex. : babiche, balancine, baliser, boîtes sauvages, boucane, ceinture fléchée, coureurs des bois, érablière, frasil, graphinier, marchement, mocassin, poudrerie, savane, totem, etc.). Avec quelle joie nous apprîmes, de la plume savante de Léon Lorrain, membre de la Société royale du Canada, qu'il existe, dans un petit village de la province de Québec, au fond d'une grande cour, un bâtiment vétuste surmonté d'une petite coupole et qu'on nomme communément le champ de la yance. Les raffinés l'appellent, pour inventer une explication, le champ de l'Alliance. En 
réalité, c'est le lieu de l' "Ö̈ance», donc de l'audience où le juge français de l'Ancien régime rendait la justice à nos ancêtres venus dans la Nouvelle-France.

Pendant que nous relevions, en France, un petit nombre de «faux amis» embarrassant la langue du Canada, faux en ce qu'ils font croire à une identité de sens ou de valeur grammaticale entre le mot français et le mot anglais d'une même forme en anglais et en français (ex. : change) ou à peu près (ex. : conservative en anglais et conservatrice en français), de petit nombre parce que nous nous en tenions aux termes de la langue juridique, l'équipe du très érudit Comité de linguistique de Radio-Canada, appliquée, comme l'avait fait notre confrère Victor Barbeau dans son ouvrage le Français du Canada, à récolter et corriger les canadianismes mal venus et inutiles, introduisait dans sa proscription les «faux amis » nés chez les francophones du Québec de l'influence anglo-américaine.

Il est peu vraisemblable qu'un élève d'un de nos collèges d'enseignement général, où l'étude de l'anglais est devenue obligatoire à peu près pour tous, s'avisera, en présence de l'expression « a school of herring», alors que le mot school qu'il rencontre à chaque instant est un ami de notre mot école, de traduire par «école de harengs». Il sentira le faux ami, consultera son dictionnaire à l'article herring et trouvera «banc de harengs » comme traduction de «school of herring ». D'où j'envisagerais un dictionnaire - il existe peut-être — qui, à la suite de chaque mot important, signalerait les mauvais canadianismes formés par abus du mot étudié en précisant : «il ne faut pas dire...; on dit... », sans même honorer d'un (fam.) ou d'un (pop.) ce qui doit être condamnation absolue.

L'inconvénient de cette méthode serait le nombre des exécutions, tant est grande la quantité de mauvais canadianismes que dénoncent les grammairiens du Canada comme locutions rendues inutiles par le français universel et contraires en même temps au sens voulu par leur auteur ou à la syntaxe. Qu'à la liste par nous abordée en matière juridique pour montrer le péril de la contamination, on ajoute tous les «faux amis »de la langue commune classés méthodiquement dans les fiches jaunes du Comité de linguistique et dans les travaux de Victor Barbeau, de Robert Dubuc et de Jean-Marie Laurence, on constate avec douleur que, malgré tant d'efforts pour aboutir à un français universel, la traduction littérale de mots anglais de forme française a produit une foule énorme d'alliances de mots impropres ou incorrectes qualifiées d'expressions courantes au Canada français. On ne comprend pas pourquoi nos cousins du Canada parlant en francais s'obstinent à appeler ingénieur (de l'anglais engineer) n'importe quel mécanicien, à appeler un estimé (de l'anglais estimate) ce que nous appelons un devis, à appeler article de seconde main (de l'anglais second hand) un article d'occasion, à baptiser vêtement de base (de l'anglais foundation garments) ce qui est lingerie de dessous!

Je m'attacherai aujourd'hui, dans le cercle de la traduction de la langue judiciaire, à l'examen de ces faux amis constitués par une alliance de mots en un français canadien utilisant un ou plusieurs mots de la locution anglaise correspondante au moyen d'une traduction littérale qui détermine une impropriété par rapport au sens recherché ou un solécisme par fautive construction grammaticale.

Soit l'expression courante au Canada : "marchandises en approbation» copiant l'anglais «merchandises on approbation», dont le sens donnerait en 
français de France : "marchandises en consignation», formule correcte quant à la propriété des termes et quant à la syntaxe, alors que la canadienne est une impropriété, le terme «approbation»n'ayant jamais eu chez nous le sens de consignation.

L'expression canadienne : «vous pouvez dépendre sur lui », copie de l'anglais : "to depend upon him», est un solécisme et un faux ami, puisque le sens de cette formule anglaise est, en français : "Vous pouvez compter sur lui. »

Les formulaires de France donnent : «Le tribunal appointe le demandeur à prouver par témoins que... » Le verbe appointer a alors le sens de autoriser, inviter. Mais, quand le vocabulaire canadien français met : «appointer un héritier », ce n'est pas à ce sens français traditionnel qu'il puise; c'est au sens anglais (to appoint = désigner). En français canadien, « appointer un héritier» est un faux ami.

Nous ne voulons pas blâmer toute alliance de mots qui, pour exprimer une idée, serait autre que la formule ordinaire. Nous ne voyons aucun inconvénient à ce que, au lieu de la forme des tribunaux de France : «jugement passé en force de chose jugée », les juridictions francophones de Belgique écrivent : «jugement coulé en force de chose jugée». Cette formule, pour nous insolite, n'est pas impropre; elle dit ce qu'il faut dire, avec le même sens que la formule de France, sans impropriété ni solécisme.

Les Canadiens disent : «un bris de contrat», locution qui a le même sens que notre « annulation de contrat» ou notre « révocation de contrat». Rien à objecter, même si c'est une copie de l'anglais (to break a contract), à plus forte raison si la locution vient de l'ancien français, comme bris de clôture, bris de scellés, bris de prison, que conserve notre langue judiciaire.

D'ailleurs, nous avons en France des provincialismes judiciaires (indulger un délai ; prendre à profit les conclusions, etc.). Ils sont peu nombreux et d'une aire géographique très limitée (cf. le Style des jugements, $4^{\mathrm{e}}$ édit., $\mathrm{n}^{\circ} 31$ ). J'ai dit qu'ils risquaient d'être mal compris des agents d'exécution. C'était faire des réserves. Mais la conjoncture est plus grave au Canada, où l'anglais se trouve en position de force. De sorte que «bris de contrat» rentrerait dans la catégorie des locutions correctes et compréhensibles mais qui ne sont pas d'usage dans le français universel et qu'il est recommandé d'écarter, par système défensif, dans les genres soutenus.

Voici enfin d'autres exemples de faux amis touchant peu ou prou à la langue judiciaire :

mettre l'emphase sur un argument (de l'anglais to lay emphasis on) pour : insister sur un argument;

il est préjugé contre vous (de l'anglais to be prejudicied) pour : il est prévenu contre vous;

il opere un commerce (de l'anglais to operate) pour : il exploite un commerce;

investiguer sur une affaire (de l'anglais to investigate) pour : enquêter sur une affaire;

une étude légale (de l'anglais legal office) pour : un cabinet (d'avocat);

canceller son testament (de l'anglais to cancel) pour : révoquer son testament;

juger une cause au mérite (de l'anglais on its merit) pour : juger au fond; 
intervenir en capacité de (de l'anglais in the capacity of) pour : en qualité de ; le prévenu a fait des admissions (de l'anglais admission) pour : le prévenu a fait des aveux; une erreur cléricale (de l'anglais clerical) pour : une faute de plume; accusé de conspiration (de l'anglais conspiration) pour : entente criminelle; billet venant à maturité (de l'anglais maturity) pour : qui vient à échéance; intérêts mutuels des demandeurs (de l'anglais mutual) pour : intérêts communs; poursuivre pour offense (de l'anglais offence) pour : délit ; faire une partition (de l'anglais partition) pour : faire un partage ; la sécurité d'un prêt (de l'anglais security) pour : garantie d'un prêt; une lettre morte (de l'anglais dead letter) pour : une lettre au rebut; le nom premier (de l'anglais first name) pour : le prénom; ordonner un ajustement (de l'anglais adjustment) pour : une expertise ; le paiement était passé dû (de l'anglais overdue) pour : le jour du paiement était échu ; il s'est objecté à cette mesure (de l'anglais to object) pour : il s'est opposé à cette mesure ; je soumets au tribunal que (de l'anglais to submit that) pour : j'allègue devant le tribunal que... ;

il m'a référé à vous (de l'anglais to refer) pour : il m'a adressé à vous ; vous pouvez dépendre sur lui (de l'anglais to depend) pour : compter sur lui ; il a disposé de ses adversaires (de l'anglais to dispose of) pour : il a vaincu ses adversaires ; cet homme est suspect (de l'anglais to suspect - et non suspicious) pour : homme méfiant!

Ainsi, l'alliance de mots «homme suspect » qui en français de France et en français universel viserait un présumé coupable, est appliquée, au Canada français, à la victime méfiante, par le maléfice du verbe anglais «to suspect»)!

Que peut faire le patriote traducteur en présence de deux générations de Québécois; l'une qui, submergée par la publicité et le style de la presse, prend la qualification d'homme suspect pour un compliment; l'autre qui voit dans cette qualification le commencement d'une action judiciaire pour délit de droit commun?

Selon moi, la situation du traducteur est moins embarrassante quand un néologisme américain, introduit brusquement dans l'intérieur de la phrase française, la rend certes cacophonique mais, justement, par cette discordance de ton, engage l'auditeur et le lecteur à bien peser le sens du tout. Autrefois, M. Jean-Paul Vallée a fourni au Comité linguistique de Radio-Canada une série de termes anglais néologiques logés, en matière de machines électriques, dans des notices de langue française. Là où les constructeurs et vendeurs n'osaient fabriquer les mots, il donnait l'exemple d'un effort de traduction individuel et magistral. Nous constatons que, sous cet essai, « le moteur lache sur sa bracket » devient, en évitant le faux ami : «le moteur joue sur son support». Mais, puisqu'il paraît que le Centre de terminologie d'Ottawa a déjà relevé plus de mille néologismes américains restant à traduire, on sent la nécessité d'un accord collectif des groupements de traducteurs et de techniciens du Canada, tel que celui qui anime le Comité d'étude des termes techniques français. Ce Comité travaille, comme notre Académie des sciences elle-même, en vue d'empêcher que la langue de leur ressort prolifère dans l'anarchie.

Pierre Mimin 